from the pre-intervention period was abstracted from clinic records. Multilevel logistic regression models with random intercepts to account for clustering within clinics were used to compare rates of syphilis case detection (number of confirmed positive cases over the total number of clinic attendees) in the 6-month periods prior to and during the intervention. Estimates were adjusted for age and sex of clinic attendees.

Results A total of 7900 patients (49.0\% male; 17.7\% under the age of 25) sought care at one of the five STD clinics over the course of the study. Adjusted odds of a positive syphilis screen were greater during the intervention period compared to the pre-intervention interval (odds ratio, 1.33; $95 \%$ confidence interval, 1.14-1.56). Variability in clinic-level effects were substantial given the small number of sites of this pilot study.

Conclusion Results of a conditional pay-for-performance pilot study demonstrate the feasibility and preliminary effectiveness of a conditional P4P strategy to improve syphilis case detection in Chinese clinical settings. Plans are underway for a fully powered randomized trial, findings from which could inform the utility of this approach for improving detection of common STDs in other resource constrained settings.

Disclosure No significant relationships.

\section{P725 FREQUENCY AND CHARACTERISTICS OF BIOLOGIC FALSE POSITIVE TESTS FOR SYPHILIS, REPORTED IN FLORIDA AND NEW YORK CITY, 2013-2017}

\footnotetext{
${ }^{1}$ James Matthias*, ${ }^{2}$ Ellen Klingler, ${ }^{3} J u l i a$ Schillinger, ${ }^{4}$ Thomas Peterman, ${ }^{5}$ Craig Wilson. ${ }^{1}$ Centers for Disease Control and Prevention, Division of STD Prevention, Tallahassee, USA; ${ }^{2}$ New York City Department of Health and Mental Hygiene, New York City, USA; ${ }^{3}$ Centers for Disease Control and Prevention, New York City, USA; ${ }^{4}$ Centers for Disease Control and Prevention, Division of STD Prevention, Atlanta, USA; ${ }^{5}$ Florida Department of Health, STD and Viral Hepatitis Section, Tallahassee, USA

\subsection{6/sextrans-2019-sti.786}

Background Biologic false positive (BFP) non-treponemal test results, defined as specimens with reactive non-treponemal (NT) and non-reactive treponemal test results are received and processed by United States public health surveillance programs. Non-treponemal BFPs can be attributed to a variety of infectious and non-infectious diseases. However, little is known about the distribution of BFP NT titers. We describe the frequency, NT titer distribution, and descriptive characteristics of people with BFP NT results reported in Florida and New York City (NYC).

Methods Reactive NTs and BFPs (reactive NT test and $\geq 1$ non-reactive treponemal tests from a person with no past or current reactive treponemal test) were extracted from Florida's and NYC's sexually transmitted diseases surveillance systems for 2013-2017. Results were de-duplicated by specimen collection date, test type, and titer value. For individuals, BFPs were stratified by site, patient characteristics, and highest reported titer during study period.

Results A total of 526,540 reactive NTs were reported. Among these were 57,850 BFPs (28,183 Florida and 29,397 NYC), $11 \%$ of all reactive NTs, from 39,920 individuals $(19,313$ Florida and 20,607 NYC). Titers of $1: 1$ accounted for $55 \%$ $(n=31,580)$ of all BFPs, but 5,250 (9\%) were $\geq 1: 8$, including $654(1.1 \%) \geq 1: 32$. Persons with BFP were most often women $68 \%(n=27,161 / 39,920)$. Individuals $40+$ years were at increased odds (OR 2.12; 95\% C.I. 1.78-2.55) of having a high titer $\mathrm{BFP}(\geq 1: 32$ titer).

Conclusion Syphilis BFPs (non-cases) account for a substantial number of reported tests that require processing. Some countries classify titers $\geq 1: 8$ as presumptive syphilis without treponemal testing, thus may over-count cases. Areas requiring both treponemal and NT tests for syphilis case reporting may benefit from requiring laboratories to report negative treponemal tests when they are associated with reactive NT tests. Review of patient histories might identify underlying conditions that contribute to high-titer BFP results.

Disclosure No significant relationships.

\section{P726 SYPHILIS - DO WE SEE THE END OF THE STEEP RISE IN CASES IN GERMANY?}

${ }^{1}$ Klaus Jansen*, ${ }^{2}$ Viviane Bremer. ${ }^{1}$ Robert Koch Institute, Infectious Disease Epidemiology, Berlin, Germany; ${ }^{2}$ Robert Koch Institute, Berlin, Germany

\subsection{6/sextrans-2019-sti.787}

Background The number of reported syphilis cases in Germany doubled between 2001-2004 to over 3,000/year and remained stable until 2009. Between 2010 and 2016, the annual increase was between $7 \%$ and $22 \%$. We analysed syphilis surveillance data to assess characteristics of current epidemiological dynamics in order to initiate appropriate prevention measures.

Methods Since 2001, laboratories are required to notify syphilis diagnoses anonymously, physicians complement clinical information. Potential double notifications are identified. Since 2016, HIV-status is reported. We analysed syphilis cases by year of diagnosis, age, sex, area of residence, and transmission category.

Results 7,520 cases were reported in 2017, corresponding to a $4.5 \%$ rise compared to 2016. Incidence was 9.1/100,000 inhabitants, with highest incidences in metropolitan cities as Cologne (39.4), Berlin (37.9), and Munich (35.0). From January to June 2018, syphilis cases dropped by $4.3 \%$ compared to this period in 2017. Men accounted for $94 \%$ of cases in 2017. $83 \%$ of cases with information on transmission route were men who have sex with men (MSM), 17\% heterosexuals. The proportion of MSM aged 40 years or above was continuously above $45 \%$ since 2007 . Syphilis reinfection was reported for 27\% HIV-negative MSM and 63\% HIV-positive MSM. HIV-coinfection was reported for 48\% MSM and 7\% heterosexuals. HCV-coinfection was reported for 7\% HIV-positive MSM and $1 \%$ of HIV-negative MSM.

Conclusion Data showed a high burden of disease in MSM in metropolitan cities and higher age groups. Increasing risk behavior due to HIV pre-exposure prophylaxis (PrEP) could impact the syphilis epidemic in Germany in specific groups, considering the high proportion of Syphilis reinfections as well as a distinct rate of HCV-coinfection. To avoid negative effects of PrEP for the Syphilis epidemic, targeted and innovative approaches to foster early screening and treatment, like internet counselling, home sampling, home testing and broadening venue-based (rapid) testing should be discussed.

Disclosure No significant relationships. 